\title{
Interleukin-6 and Cardiovascular Diseases
}

\author{
Tsugiyasu KANDA, ${ }^{1}$ MD and Takashi TAKAHASHI, ${ }^{1}$ MD
}

\begin{abstract}
SUMMARY
Inflammatory cytokines are important for both cardiovascular scientists and practicing clinicians. Interleukin-6 (IL-6) has been emphasized by reports of elevated circulating as well as intracardiac IL-6 levels in patients with congestive heart failure (CHF). IL-6 may contribute to the progression of myocardial damage and dysfunction in chronic heart failure syndrome resulting from different causes. As the cause of CHF in cardiomyopathy, myocarditis, allograft rejection, and left ventricular assist device (LVADs) conditions, circulating IL-6 levels are associated with the severity of left ventricular dysfunction, and are also strong predictors of subsequent clinical outcomes. Continuous and excessive production of IL- 6 promotes myocardial injury by breaking down both cytokine networks and viral clearance under viral myocarditis. Although IL-6 is likely important in the process of viral antigen presentation, early activation of immune responses and attenuation of viral replication also appear to be significant in an animal model of viral myocarditis. IL-6 can cause cardiac hypertrophy through the IL-6 signal transducing receptor component, glycoprotein 130. There are several interesting cases of cardiac myxoma complicated with mediastinal lymphadenopathy or left ventricular hypertrophy. Increased expression of IL6 is observed in the myocardium of all donor hearts showing marked dysfunction. Myocardial IL-6 concentrations are also significantly higher in LVAD candidates compared with advanced heart failure patients. Although the IL-6 family plays a central role in the pathophysiology of cardiovascular diseases, it remains to be determined whether the IL6 family is beneficial or detrimental. Future study will be needed to resolve this question. (Jpn Heart J 2004; 45: 183-193)
\end{abstract}

Key words: Interleukin-6, Coronary artery disease, Myocarditis, Cardiomyopathy

INFLAMMATORY cytokines have recently become important for both cardiovascular scientists and practicing clinicians. Locally acting cytokines play a role not only in the pathogenesis of atherosclerosis, but also in the cardiac dysfunction that accompanies systemic sepsis, viral myocarditis, and cardiac allograft rejection. ${ }^{1,2)}$ During the past decade, Parrillo and colleagues ${ }^{3,4)}$ have used intact animals and in vitro isolated heart cell preparations to systematically investigate the factors that contribute to myocardial depression in the systemic inflammatory response syndrome. Interest in the soluble inflammatory mediator, interleukin-6

From the ${ }^{1}$ Department of General Medicine, Kanazawa Medical University, Ishikawa, Japan.

Address for correspondence: Tsugiyasu Kanda, MD, Department of General Medicine, Kanazawa Medical University, 1-1 Daigaku, Uchinada-machi, Kahoku-gun, Ishikawa 920-0293, Japan.

Received for publication September 24, 2003.

Accepted October 24, 2003. 
(IL-6), has been amplified by reports of elevated circulating as well as intracardiac IL-6 levels in patients with congestive heart failure (CHF) ${ }^{5-7)}$ Elevated levels of circulating IL-6 were reported even in CHF patients with normal levels of other cytokines, such as tumor necrosis factor-alpha (TNF- $\alpha$ ). It is now significant attention to discuss the IL-6 levels in patients with CHF.

Structure and function of interleukin-6: IL-6, a 185 amino acid polypeptide, is refolded. One hundred and seven out of 185 amino acid residues are well defined in the final structure, with $18 \mathrm{~N}$-terminal and $8 \mathrm{~A}-\mathrm{B}$ loop amino acids displaying no interpretable electron density. The three-dimensional structure has been used to construct a model of IL-6 interacting with the IL-6 receptor (IL-6R) (-chain) and glycoprotein 130 (gp130) (-chain) that gives new insight into the process of molecular recognition and signaling with a functional hexameric IL-6 receptor complex. ${ }^{8)}$

IL-6 is a pleiotropic cytokine with a variety of biological activities, including the ability to stimulate B-cell differentiation, ${ }^{9)}$ activate thymocytes and T-cells for differentiation ${ }^{10)}$ activate macrophages, ${ }^{11)}$ stimulate hepatocytes to produce acute-phase proteins, ${ }^{12)}$ and activate natural killer (NK) cells. ${ }^{13)}$ IL-6 also possesses anti-inflammatory properties. ${ }^{14)}$ IL-6 would be expected to produce a restorative effect by inducing the activity of immunoregulatory cells in the replication setting.

Interleukin-6 in cardiovascular diseases: IL-6 has both pro-inflammatory and anti-inflammatory properties. It is produced not only by immune cells and immune accessory cells including monocytes and macrophages, but also by cardiovascular components, such as endothelial cells, vascular smooth-muscle cells, and ischemic myocytes. ${ }^{15-18)}$ What makes IL-6 particularly interesting to physicians is its involvement not only with inflammation but also with the regulation of cardiac metabolism (Figure 1).

Interleukin-6 and C-reactive protein: C-reactive protein (CRP) evokes the production of IL-6. Additional evidence to support a role for IL- 6 as a mediator of the actions of CRP is provided by experiments examining the effects of IL-6 inhibition. Anti-IL-6 antibodies attenuated CRP-mediated expression of adhesion molecules, monocyte chemoattractant protein-1 secretion, and macrophage uptake for low-density lipoprotein. ${ }^{19)}$

CRP stimulates the production of IL-6 concurrently in a parallel fashion. Alternatively, it is possible that CRP quenches an unidentified inhibitory factor, such as nitric oxide, which is known to decrease IL-6 expression. It is also plausible that CRP serves to activate nuclear factor-B (NF-B), with the resultant increase in expression of IL-6 and other NF-B-regulated systems. However, this remains to be determined. 


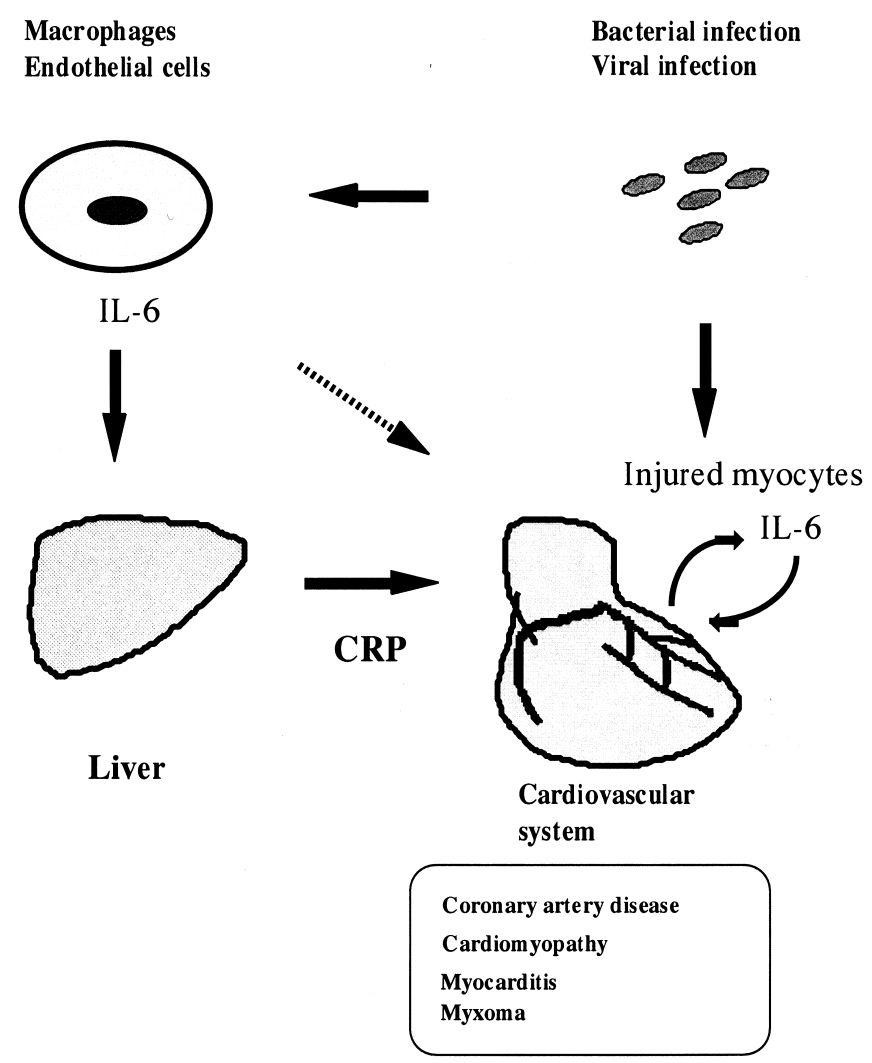

Figure 1. Relationships between interleukin-6 (IL-6), C-reactive protein (CRP), and cardiovascular diseases.

IL-6 is a key inflammatory factor that has been implicated in the pathogenesis and clinical course of atherosclerotic vascular disease. ${ }^{20)}$ IL-6 is known to be secreted from several cell types, including endothelial cells, macrophages, lymphocytes, and adipocytes, and exerts its biological actions through a complex yet well-defined fashion. The IL-6 receptor complex consists of two membranebound glycoproteins, an $80 \mathrm{kD}$ ligand-binding component (termed IL-6R) and a $130 \mathrm{kD}$ signal-transducing component (termed gp130). ${ }^{20)}$ Although IL-6 may bind the IL-6R and elicit a biological response, it also activates a soluble IL-6R (sIL-6R). The activated IL-6/sIL-6R complex serves as a potent agonist that binds the signal-transducing component of the membrane-bound receptor, gp130, with high affinity. Through this mechanism, IL-6 is believed to potentiate its own biological activity and exert effects in cells that lack the IL-6R per se. Indeed, the 
IL-6/sIL-6R complex has been demonstrated to stimulate leukocyte recruitment and to promote inflammatory responses by endothelial cells. ${ }^{21,22)}$

The aforementioned description assumes importance because a recent study has demonstrated that CRP is a physiological regulator of sIL-6R shedding in human neutrophils and markedly increases formation of the sIL-6R/IL-6 complex. ${ }^{21)}$ Data from this study suggest that CRP may also function to increase IL-6 secretion from endothelial cells. By stimulating endothelial production of IL-6 and neutrophil sIL-6R shedding, CRP may serve to markedly exaggerate the actions of IL- 6 at the site of the endothelium. Since IL- 6 is a potent stimulus for CRP expression in liver, the increased vascular production of IL-6 may represent a positive feedback mechanism for the continued production of CRP from the liver.

Interleukin-6 and coronary heart disease: Recently, increased levels of circulating IL-6 and CRP have been also associated with high risk of all-cause mortality in older people. ${ }^{7,23)}$ Serum levels of IL-6, along with other cytokines, have also been associated with unfavorable clinical outcomes in patients hospitalized for unstable angina. ${ }^{24)}$ IL-6 concentrations are associated not only with the severity of the conditions, but are also very strong predictors of subsequent outcomes. For example, Biasucci, et $a l^{24)}$ demonstrated that the patients with unstable angina and a complicated in-hospital course have higher IL-6 levels than subjects without complications. Pro-inflammatory cytokines may be involved in destabilizing and disrupting atherosclerotic plaque in coronary arteries. The presence of an inflammatory process characterizes the site of plaque rupture or erosion, ${ }^{25)}$ and the pro-inflammatory cytokines up-regulate the expression of matrix metalloproteinases, which are known to be involved in the vascular remodeling and plaque disruption. $^{26)}$

Interleukin-6 and congestive heart failure: Elevated levels of circulating cytokines have been demonstrated in patients with heart failure. In particular, IL-6 concentrations were related to the severity of left ventricular dysfunction and to the degrees of activation of the sympathetic and renin-angiotensin systems. ${ }^{27)}$ The raised levels of IL-6 correlate with decreased cardiac functional class, lowered ejection fraction, and poor prognosis. ${ }^{7,28-30)}$ A recent study has shown increased myocardial IL- 6 expression is associated with the progression of heart failure. ${ }^{31)}$ IL-6 may be a good indicator of the deterioration.

Although previous studies have examined the effects of acute hemodynamic pressure overloading on pro-inflammatory cytokine gene expression, the influence of sustained hemodynamic overloading has not been examined. Continuous hemodynamic overloading provokes a transient increase in both pro-inflammatory cytokine and cytokine receptor gene expression. However, the decrease in pro-inflammatory cytokine gene expression occurred under the absence of 
changes in the loading conditions, suggesting that the expression of pro-inflammatory cytokines in the heart is regulated, at least in part, by both load-dependent and load-independent mechanisms. ${ }^{32)}$ It is not clear whether this increase of circulating cytokines is mainly an epiphenomenon of the severity of the underlying cardiac impairment, ${ }^{33)}$ but in vitro and in vivo investigations suggest that the proinflammatory cytokines might depress myocardial contractility. ${ }^{34)}$

In a previous report we showed that the expression of IL-6 and the coexpressed ANP in IL-6-positive myocardium were observed in heart from autopsy cases of acute myocardial infarction. ${ }^{18)}$ Interestingly, the elevation of ANP and BNP mRNA expression in heart from IL-6 transgenic mice did not occur regardless of myocardial impairment (Figure 2). We believe that the mismatch of natriuretic peptide expression may be induction failure caused by IL-6 overexpression. These results suggest that the continuous expression of IL- 6 elicits undisclosed overloading and induces the transcription of natriuretic peptides in heart, and that the induction failure of natriuretic peptides during the CHF shows impaired adaptation to myocardial injury.
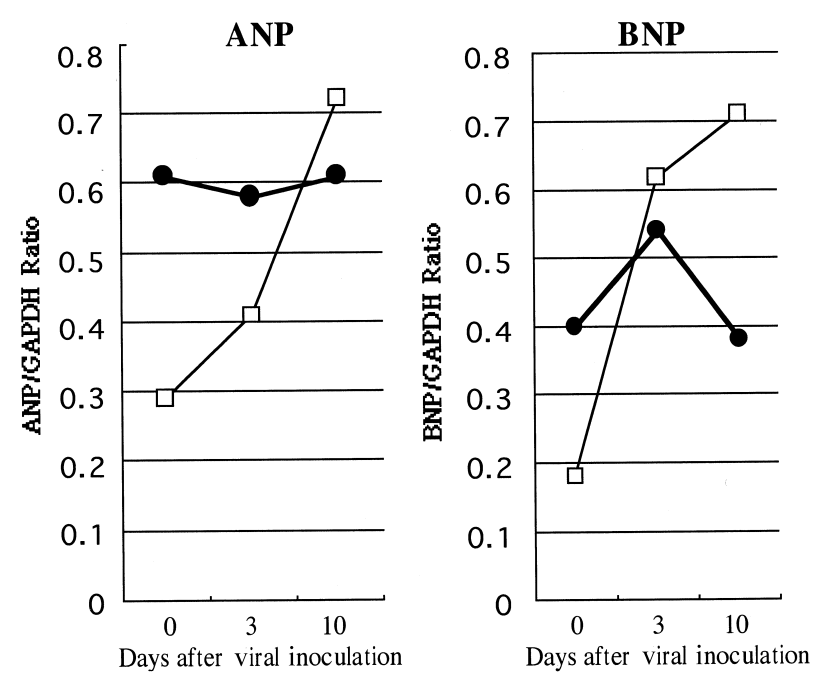

;IL-6 Transgenic mice

$\square$;Wild type mice

Figure 2. Variations of atrial natriuretic peptide (ANP) and B-type natriuretic peptide (BNP) mRNA expression in hearts from interleukin-6 (IL-6) transgenic mice or wild type mice after inoculation of encephalomyocarditis virus. The comparative gene expression levels of ANP (left) and BNP (right) relative to glyceraldehyde-3-phosphate dehydrogenase (GAPDH) are shown. 
Interleukin-6 in myocarditis: IL-6 reduces myocardial damage in mice with viral myocarditis. In addition, it stimulates differentiation of B cells, macrophages, and NK cells in vivo. ${ }^{15,35-37)}$ IL-6 under encephalomyocarditis virus-induced myocarditis in mice modulated early immune responses responsible for B cells, macrophages, and NK cells. Modification of the immune responses together with a reduction in viral replication seems to contribute to the mechanism of the IL-6 effect. Although IL-6 is likely important in the process of viral antigen presentation, early activation of immune responses and attenuation of viral replication also appear to be significant. The direct approaches to elucidate the role of IL-6 in inflammatory and immunity are to use genetically engineered transgenic mice. Overexpression of IL- 6 in transgenic mice results in massive plasmacytosis and hypergammaglobulinemia. ${ }^{38)}$ Our results showed that the continuous and excessive production of IL-6 promotes myocardial injury through a breaking down of both cytokine networks and viral clearance. ${ }^{39)}$ These experiments indicate that IL6 has a beneficial effect in early inflammatory stages, but the constitutive activation of IL- 6 accelerates myocardial damage in the process of viral infection.

Interleukin-6 in infective endocarditis: It was hypothesized that assessment of circulating IL-6 and interleukin-2 receptor (IL-2R) levels may provide new diagnostic criteria for inflammation in infective endocarditis (IE). Serum concentrations of IL-6 and IL-2R were decreased continuously during antibiotic treatment. Thus, these cytokines can be suitable for monitoring the course of inflammation during treatment for IE. ${ }^{40)}$

On the other hand, serum IL- $1 \alpha$ and TNF- $\alpha$ levels were not affected in the process of IE. A further understanding of the role of serum cytokine concentrations in the diagnosis, treatment, monitoring, and prognosis for IE may be valuable under the status of suspected diagnosis, especially when the pathogens cannot be detected in blood cultures. ${ }^{41)}$

Interleukin-6 in sepsis: IL-6 is known to be an important pro- and anti-inflammatory cytokine, which is up-regulated under the condition of sepsis. In the early phases of sepsis, the activated complement product, $\mathrm{C} 5 \mathrm{a}$, plays a harmful role in rodents undergoing cecal ligation puncture (CLP) or infusion of lipopolysaccharide. IL-6 strongly promotes expression of $\mathrm{C} 5 \mathrm{a}$ receptors in rat lung epithelial cells, thymocytes, and endothelial cells. ${ }^{42,43)}$ Blockade of IL-6 expression during CLP-induced sepsis in mice causes a significantly decreased induction of C5a receptors in several organs including lung, liver, kidney, and heart, leading to improved survival. ${ }^{44}$

Interleukin-6 and cardiac hypertrophy: Transgenic mice overexpressing either IL-6 or IL-6R alone did not show detectable myocardial abnormalities. Neonatal cardiac muscle cells from normal mice, when cultured in vitro, were enlarged in response to a combination of IL-6 and a soluble form of IL-6R. The results sug- 
gest that activation of the gp130 signaling pathways leads to cardiac hypertrophy, and that this signaling may be involved in physiological regulation of the myocardium. ${ }^{45)}$

Clinical recognition of the extent of ventricular hypertrophy is a strong predictor of adverse events. Accordingly, identification of the signals that mediate the pathways from mechanical stress to downstream cellular events has been a major area of interest. Both cardiac myocytes and nonmyocytes are direct biomechanical sensors of hemodynamic pressure loading. Growth signals are generated by the release of growth factors and cytokines, which lead to a localized response. The factors that have been implicated in this response include IL-6-related cytokines (cardiotrophin 1). ${ }^{46,47)}$ We previously described that overexpression of IL-6 was confirmed in the injured myocardium with hypertrophy in patients who died of acute myocardial infarction (AMI) within seven days after onset. The expression of IL-6 in the myocardium under AMI appears to be associated with the pathogenesis of cardiac hypertrophy. ${ }^{18)}$

Interleukin-6 and cardiac myxoma: Cardiac myxoma originally produces IL6. ${ }^{48,49)}$ We experienced a patient presenting with disappearance of mediastinal lymphadenopathy after resection of a massive cardiac myxoma, which by itself secreted IL-6. The pathogenesis of cardiac myxoma-associated lymphadenopathy can be explained by IL- 6 production in the cardiac myxoma.

Cardiac myxoma often causes embolization into the systemic circulation, and fragments coming out of this tumor sometimes continue to grow at many embolized sites. ${ }^{50)}$ IL-6 is also known to be one of the angiogenic factors when the development of tumor local growth and distant metastasis. ${ }^{50)}$ Overproduction of these angiogenic inducers may account for the clinical features of cardiac myxomas, such as frequent recurrence and distant metastasis. ${ }^{49,50)}$ These materials can act as autocrine growth factors of myxoma cells, as shown in other kinds of human tumor cell lines. Therefore, IL-6 production by an atrial myxoma is an important factor leading to intravascular metastasis or embolization. In addition, IL-6 secreted from a myxoma may contribute to the pathogenesis of ventricular hypertrophy in the patient. There are several interesting cases of cardiac myxoma complicated by either mediastinal lymphadenopathy or left ventricular hypertrophy. ${ }^{51,52)}$ These cases may suggest a clinical role for IL- 6 in the heart.

Interleukin-6 in heart transplantation: Myocardial dysfunction in the donor heart is a common problem and is so severe as to preclude its use for transplantation in $20 \%$ of cases. Brain death has been shown to result in various degrees of myocardial dysfunction. To assess the potential of the donor heart to respond to IL6, some studies have investigated the expression of IL-6R components in the myocardium of donor hearts before transplantation. Donor heart dysfunction early after transplantation has been associated with the cytokine storm after donor 
brain death. Pro-inflammatory cytokines are thought to play a critical role in this process. IL-6 is especially interesting since it has contributed to cardiac allograft dysfunction and is related to an impaired prognosis. Its action requires co-expression of specific IL6R and the common signal transducer of the IL6 family gp130 in the donor hearts. Messenger RNA of IL6, IL6R, and gp130 was strongly expressed in all chambers of the donor hearts, whereas the right ventricles of control patients showed no expression. Right ventricles of failing hearts showed levels of IL6, IL6R, and gp130 mRNA that were comparable with those found in the donor hearts. While IL6 was mainly expressed in the myocytes, both receptor components were preferentially observed mainly in the interstitial cells. The expression of IL6 receptor components in the donor heart before transplantation establishes the condition sine qua non for the response of the donor heart to circulating IL6. This understanding can lead to the explanation of association between elevated serum IL6 concentrations and acute cardiac allograft dysfunction in the early postoperative period. ${ }^{53)}$ Myocardial and serum IL-6 levels from donors with myocardial dysfunction (unused donors), from donors with good ventricular function (used donors), and from patients with advanced heart failure were examined. Increased expression of IL-6 in the myocardium of all donor hearts showing marked dysfunction was found. IL-6 mRNA expression was higher in the unused donor hearts than in the donor hearts used for transplantation. Elevated levels of IL-6 mRNA in the unused donor hearts compared with the hearts of patients with advanced failure were observed. IL- 6 mRNA levels in the used donor hearts were higher than in the hearts of advanced failure. Similar findings in the serum were confirmed. These events may have important therapeutic implications. ${ }^{54)}$ Inhibition of IL-6 secretion in cardiac transplant recipients is an important factor to protect against the development of cardiac allograft vasculopathy. HMG-COA reductase is reported to inhibit IL-6 secretion. ${ }^{55)}$

Interleukin-6 and left ventricular assist devices: Left ventricular assist devices (LVADs) have become an established treatment for patients with severe heart failure. The molecular mechanisms underlying the decompensation of heart failure remain poorly understood. Expression levels of IL-6 mRNA in myocardium at the time of LVAD implantation were higher than under the stable condition of advanced heart failure. Understanding the mechanisms involved may be useful to decide the timing of LVAD implantation and to identify new therapeutic targets for advanced heart failure. ${ }^{56)}$ Myocardial IL-6 concentrations in LVAD candidates were significantly higher than in patients with advanced heart failure. Significantly elevated levels of myocardial IL-6 were observed for cases who died after LVAD implantation compared with for those who survived over 1 year after implantation. Thus, IL-6 may be a beneficial indicator of the prognosis in the LVAD candidates, and may be involved in the pathologically deteriorated status, 
although the elevation of IL-6 levels in dead patients could imply an irreversible condition.

Future directions of interleukin-6 for cardiovascular diseases: Cytokines including IL-6, TNF- $\alpha$, and IL-1 are responsible for the development and progression of cardiovascular diseases. Some clinical trials have been conducted in patients with cardiovascular diseases. ${ }^{57)}$ Although the IL-6 family plays a key role in the pathophysiology of cardiovascular diseases, it remains to be determined whether the IL- 6 family is beneficial or detrimental. Future studies will be needed to resolve this question.

\section{ACKNOWLEDGMENT}

Preparation of this review article was supported in part by the Science Research Promotion Fund of the Promotion and Mutual Aid Corporation for Private Schools of Japan (Dr. T. Kanda).

\section{REFERENCES}

1. Blum A, Miller H. Pathophysiological role of cytokines in congestive heart failure. Annu Rev Med 2001; 52: $15-27$.

2. Wollert KC, Drexler H. The role of interleukin-6 in the failing heart. Heart Fail Rev 2001; 6: 95-103.

3. Parrillo JE. Pathogenetic mechanism of septic shock. N Engl J Med 1993; 328: 1471-7.

4. Kumar A, Thota V, Dee L, et al. Tumor necrosis factor- and interleukin- $1 \beta$ are responsible for in vitro myocardial cell depression induced by human septic shock serum. J Exp Med 1996; 183: 949-58.

5. MacGowan GA, Mann DL, Kormos RL, et al. Circulating interleukin-6 in severe heart failure. Am J Cardiol 1997; 79: 1128-31.

6. Roig E, Orus J, Pare C, et al. Serum interleukin-6 in congestive heart failure secondary to idiopathic dilated cardiomyopathy. Am J Cardiol 1998; 82: 688-90.

7. Tsutamoto T, Hisanaga T, Wada A, et al. Interleukin- 6 spillover in the peripheral circulation increases with the severity of heart failure, and the high plasma level of interleukin- 6 is an important prognostic predictor in patients with congestive heart failure. J Am Coll Cardiol 1998; 31: 391-8.

8. Somers W, Stahl M, Seehra JS. A crystal structure of interleukin 6: implications for a novel mode of receptor dimerization and signaling. EMBO J 1997; 16: 989-97.

9. Hirano T, Yasukawa K, Harada H, et al. Complementary DNA for a novel human interleukin (BSF-2) that induces B lymphocytes to produce immunoglobulin. Nature 1986; 324: 73-7.

10. Lots M, Jirik F, Kabouridis R, et al. BSF-2/IL-6 is costimulant for human thymocytes and T-lymphocytes. J Exp Med 1988; 140: 508-13.

11. Mule JJ, McIntosh JK, Joblons DM, Rosenberg SA. Antitumor activity of recombinant interleukin-6 in mice. J Exp Med 1990; 171: 629-35.

12. Gauldie JC, Richards C, Harnich D, Lansdrop P, Baumann H. Interferon s2/BSF-2 shares identity with monocyte-derived hepatocyte stimulating factor (HSF) and regulates the major acute phase protein response in liver cells. Proc Natl Acad Sci USA 1987; 84: 7251-6.

13. Luger TA, Krutmann J, Kirnbaner R, et al. IFN-s2/IL-6 augments the activity of human natural killer cells. J Immunol 1989; 143: 1206-10.

14. Kox WJ, Volk T, Kox SN, Volk HD. Immunomodulatory therapies in sepsis. Intensive Care Med 2000; 26(Suppl 1): S124-8.

15. Mesri M, Altieri DC. Endothelial cell activation by leukocyte microparticles. J Immunol 1998; 161: 4382-7. 
16. Loppnow H, Libby P. Proliferating or interleukin 1-activated human vascular smooth muscle cells secrete copious interleukin 6. J Clin Invest 1990; 85: 731-8.

17. Gwechenberger M, Mendoza LH, Youker KA, et al. Cardiac myocytes produce interleukin-6 in culture and in viable border zone of reperfused infarctions. Circulation 1999; 99: 546-51.

18. Kaneko K, Kanda T, Yokoyama T, et al. Expression of interleukin- 6 in the ventricles and coronary arteries of patients with myocardial infarction. Res Commun Mol Pathol Pharmacol 1997; 97: 3-12.

19. Verma S, Li SH, Badiwala MV, et al. Endothelin antagonism and interleukin-6 inhibition attenuate the proatherogenic effects of C-reactive protein. Circulation 2002; 105: 1890-6.

20. Yudkin JS, Kumari M, Humphries SE, et al. Inflammation, obesity, stress and coronary heart disease: is interleukin-6 the link? Atherosclerosis 2000; 148: 209-14.

21. Modur V, Li Y, Zimmerman GA, et al. Retrograde inflammatory signaling from neutrophils to endothelial cells by soluble interleukin-6 receptor. J Clin Invest 1997; 100: 2752-6.

22. Romano M, Sironi M, Toniatti C, et al. Role of IL-6 and its soluble receptor in induction of chemokines and leukocyte recruitment. Immunity 1997; 6: 315-25.

23. Munger MA, Johnson B, Amber IJ, Callahan KS, Gilbert EM. Circulating concentrations of proinflammatory cytokines in mild or moderate heart failure secondary to ischemic or idiopathic dilated cardiomyopathy. Am J Cardiol 1996; 77: 723-7.

24. Biasucci LM, Liuzzo G, Fantuzzi G, et al. Increasing levels of interleukin (IL)-1Ra and IL-6 during the first 2 days of hospitalization in unstable angina are associated with increased risk of in-hospital coronary events. Circulation 1999; 99: 2079-84.

25. van der Wal AC, Becker AE, van der Loos CM, et al. Site of intimal rupture or erosion of thrombosed coronary atherosclerotic plaques is characterized by an inflammatory process irrespective of the dominant plaque morphology. Circulation 1994; 89: 36-44.

26. Rajavashisth TB, Xu XP, Jovinge S, et al. Membrane type 1 matrix metalloproteinase expression in human atherosclerotic plaques: evidence for activation by proinflammatory mediators. Circulation 1999; 99: 3103-9.

27. Raymond RJ, Dehmer GJ, Theoharides TC, Deliargyris EN. Elevated interleukin-6 levels in patients with asymptomatic left ventricular systolic dysfunction. Am Heart J 2001; 141: 435-8.

28. Birks EJ, Yacoub MH. The role of nitric oxide and cytokines in heart failure. Coronary Artery Dis 1997; 8: 389-402.

29. Lommi J, Pulkki P, Koskinen P, et al. Haemodynamic, neuroendocrine and metabolic correlates of circulating cytokine concentrations in congestive heart failure. Eur Heart J 1997; 18: 1620-5.

30. Kubota T, Miyagishima M, Alvarez RJ, et al. Expression of proinflammatory cytokines in the failing human heart: comparison of recent-onset and end-stage congestive heart failure. J Heart Lung Transplant 2000; 19: $819-24$.

31. Plenz G, Song ZF, Reichenberg S, et al. Left-ventricular expression of interleukin-6 messenger-RNA higher in idiopathic dilated than in ischemic cardiomyopathy. Thorac Cardiovasc Surg 1998; 46: 213-6.

32. Baumgarten G, Knuefermann P, Kalra D, et al. Load-dependent and -independent regulation of proinflammatory cytokine and cytokine receptor gene expression in the adult mammalian heart. Circulation 2002; 105 : 2192-7.

33. Deng MC, Erren M, Lutgen A, et al. Interleukin-6 correlates with hemodynamic impairment during dobutamine administration in chronic heart failure. Int J Cardiol 1996; 57: 129-34.

34. Finkel MS, Oddis CV, Jacob TD, Watkins SC, Hattler BG, Simmons RL. Negative inotropic effects of cytokines on the heart mediated by nitric oxide. Science 1992; 257: 387-9.

35. Kanda T, McManus JE, Nagai R, et al. Modification of viral myocarditis in mice by interleukin-6. Circ Res 1996; 78: 848-56.

36. Wong GG, Clark SC. Multiple actions of interleukin-6 within a cytokine network. Immunol Today 1988; 9: 137-9.

37. Puri PK, Lenard P. Systemic administration of recombinant interleukin-6 in mice induces proliferation of lymphoid cells in vivo. Lymphokine Res 1992; 11: 133-9.

38. Suematsu S, Matsuda T, Aozasa K, et al. IgG1 plasmacytosis in interleukin 6 transgenic mice. Proc Natl Acad Sci USA 1989; 86: 7547-51.

39. Tanaka T, Kanda T, McManus BM, et al. Overexpression of interleukin-6 aggravates viral myocarditis: impaired increase in tumor necrosis factor-alpha. J Mol Cell Cardiol 2001; 33: 1627-35. 
40. Alter P, Hoeschen J, Ritter M, Maisch B. Usefulness of cytokines interleukin-6 and interleukin-2R concentrations in diagnosing active infective endocarditis involving native valves. Am J Cardiol 2002; 89: 1400-4.

41. Rawczynska-Englert I, Hryniewiecki T, Dzierzanowska D. Evaluation of serum cytokine concentrations in patients with infective endocarditis. J Heart Valve Dis 2000; 9: 705-9.

42. Riedemann, NC, Guo RF, Laudes IJ, et al. C5a receptor and thymocyte apoptosis in sepsis. FASEB J 2002; 16 : $887-8$.

43. Riedemann, NC, Guo RF, Sarma VJ, et al. Expression and function of the C5a receptor in rat alveolar epithelial cells. J Immunol 2002; 168: 1919-25.

44. Riedemann NC, Neff TA, Guo RF, et al. Protective effects of IL-6 blockade in sepsis are linked to reduced c5a receptor expression. J Immunol 2003; 170: 503-7.

45. Hirota H, Yoshida K, Kishimoto T, Taga T. Continuous activation of gp130, a signal-transducing receptor component for interleukin 6-related cytokines, causes myocardial hypertrophy in mice. Proc Natl Acad Sci USA 1995; 92: 4862-6.

46. Wollert KC, Taga T, Saito M, et al. Cardiotrophin-1 activates a distinct form of cardiac muscle cell hypertrophy: assembly of sarcomeric units in series via gp130/leukemia inhibitory factor receptor-dependent pathways. J Biol Chem 1996; 271: 9535-45.

47. Sheng Z, Knowlton K, Chen J, Hoshijima M, Brown JH, Chien KR. Cardiotrophin 1 (CT-1) inhibition of cardiac myocyte apoptosis via a mitogen-activated protein kinase-dependent pathway: divergence from downstream CT-1 signals for myocardial cell hypertrophy. J Biol Chem 1997; 272: 5783-91.

48. Kanda T, Umeyama S, Sasaki A, et al. Interleukin-6 and cardiac myxoma. Am J Cardiol 1994; 74: 965-7.

49. Reynen K. Cardiac myxomas. N Engl J Med 1995; 333: 1610-7.

50. Wada A, Kanda T, Hayashi R, Imai S, Suzuki T, Murata K. Cardiac myxoma metastasized to the brain: Potential role of endogenous interleukin-6. Cardiology 1993; 83: 208-11.

51. Takizawa T, Sumino H, Kanda T, Kobayashi I, Nagai R, Ichikawa S. An interleukin-6-producing cardiac myxoma associated with mediastinal lymphadenopathy. Cardiology 1999; 92: 275-7.

52. Kanda T, Nakajima T, Sakamoto H, Suzuki T, Murata K. An interleukin-6 secreting myxoma in a hypertrophic left ventricle. Chest 1994; 105: 962-3.

53. Plenz G, Eschert H, Erren M, et al. The interleukin-6/interleukin-6-receptor system is activated in donor hearts. J Am Coll Cardiol 2002; 39: 1508-12.

54. Birks EJ, Burton PB, Owen V, et al. Elevated tumor necrosis factor-alpha and interleukin-6 in myocardium and serum of malfunctioning donor hearts. Circulation 2000; 102(Suppl 3): III352-8.

55. Weis M, Pehlivanli S, Meiser BM, von Scheidt W. Simvastatin treatment is associated with improvement in coronary endothelial function and decreased cytokine activation in patients after heart transplantation. $\mathrm{J}$ Am Coll Cardiol 2001; 38: 814-8.

56. Birks EJ, Latif N, Owen V, et al. Quantitative myocardial cytokine expression and activation of the apoptotic pathway in patients who require left ventricular assist devices. Circulation 2001; 104(Suppl 1): 1233-40.

57. Baumgarten G, Knuefermann P, Mann DL. Cytokines as emerging targets in the treatment of heart failure. Trends Cardiovasc Med 2000; 10: 216-23. 